\title{
Diagnostic problems and emergency cardiac surgery in cut-like penetrating cardiac trauma
}

\author{
Artur Bartoszcze ${ }^{1}$, Daniel J. Rams², Grzegorz Filip ${ }^{1}$, Krzysztof Bartuś ${ }^{3}$, Bogusław Kapelak ${ }^{3}$, \\ Radosław Litwinowicz ${ }^{3}$ \\ ${ }^{1}$ Department of Cardiovascular Surgery and Transplantology, John Paul II Hospital, Krakow, Poland \\ 2Jagiellonian University Medical College, Krakow, Poland \\ ${ }^{3}$ Department of Cardiovascular Surgery and Transplantology, Institute of Cardiology, Jagiellonian University Medical College, \\ Krakow, Poland
}

Kardiochirurgia i Torakochirurgia Polska 2021; 18 (3): 192-194

Penetrating cardiothoracic injuries have occurred in large numbers throughout the world from ancient times to the present. A small percentage of them, but the most severe, are penetrating cardiac traumas. Fortunately, in the $21^{\text {st }}$ century, it is possible to treat these types of injuries. Stab wounds are the most common cause of penetrating cardiac trauma, interchangeable with gunshot wounds [1-4]. They are more common in males around the age of 30 (second to fourth decade of life according to the cited study) [1-3]. Ventricular injuries account for approximately $60-70 \%$ of all cardiac injuries, with the right ventricle being slightly more commonly affected than the left due to its anatomical location $[3,5]$. The standard diagnostic methods include focused assessment sonography for trauma (FAST), cardiac echocardiogram (ECHO), multi-slice scan computed tomography (CT) [2, 3] and chest X-ray (CXR) [5, 6]. Hemodynamic instability indicates immediate surgery, while penetrating trauma should be considered in a stable patient with conservative therapy $[3,5]$. The access methods of choice in the management of these patients should be median sternotomy or anterolateral thoracotomy $[1,2$, 6], pericardiotomy [5] with possible early pericardiocentesis for evacuation of cardiac tamponade depending on the patient's condition [3]. Pericardial tamponade and hemothorax were common intraoperative findings in penetrating cardiac trauma [2]. In addition, recognizable signs of life on arrival at the hospital combined with early surgical intervention increase the chances of survival [2, 3]. In the literature review by Pereira et al. [1], the mortality rate due to penetrating cardiac trauma varies from $15 \%$ to $40 \%$ worldwide but reaches $70 \%$ in patients who were not transported to hospital according to Kaljusto et al. [3] or $94 \%$ in the study by Campbell et al. [4]. The immediate causes of death in penetrating cardiac trauma may be three basic factors: hemorrhage, cardiac tamponade or interruption of the excitation conduction mechanism [5] and long-term causes of death such as embolism, infarction, thrombosis or sepsis may also occur as complications $[1,5]$. Therefore, the management of a patient with penetrating cardiac trauma is difficult and requires immediate clinical diagnosis and treatment, which has been highlighted as "essential" by Furukawa [6].

In November 2020, a 41-year-old man was admitted to the Emergency Department (ED). The man was a foreign construction worker. According to the person who brought him to the hospital, the man had fallen on a metal rod or metal plate at the construction site. The patient had reported stabbing pains in his chest. Detailed medical questioning was not possible due to the language barrier. The patient was circulatory and respiratory stable, in good general condition with a thoracic stab wound. Vital signs presented as $36.7^{\circ} \mathrm{C}, 107 \mathrm{bpm}$ heart rate (HR), blood pressure (BP) $147 / 80 \mathrm{~mm} \mathrm{Hg}$ and $100 \%$ oxygen saturation ( $\left.\mathrm{SpO}_{2} \%\right)$. An open wound approximately $2 \mathrm{~cm}$ in size without active bleeding was visible on the left side of the chest, medial and inferior to the areola. On auscultation, bilateral bladder respiration was noted. The abdomen was soft and painless. Abnormalities in the patient's laboratory blood test showed increased inflammatory markers, elevated levels of D-dimers (DD) along with significant presence of myocardial damage markers such as creatine kinase-MB (CK-MB) and troponin I (TnI). Posterior-anterior (PA) CXR was performed. An abnormal elongated, pointed opacity was found within the Th8-Th11 vertebral bodies, possibly entering the area of the right ventricle. The object was bounded by the right paraspinal line and the anatomical left parasternal line. No evidence of pneumothorax or hemothorax was noted. Widening of the cardiac silhouette was noted (Groedel's heartto-lung ratio: 0.66$)$. The patient underwent a median sternotomy with pericardiotomy. Despite the presence of fluid, no signs of cardiac tamponade (cardiac compression) were found. The man was operated on using extracorporeal cir- 

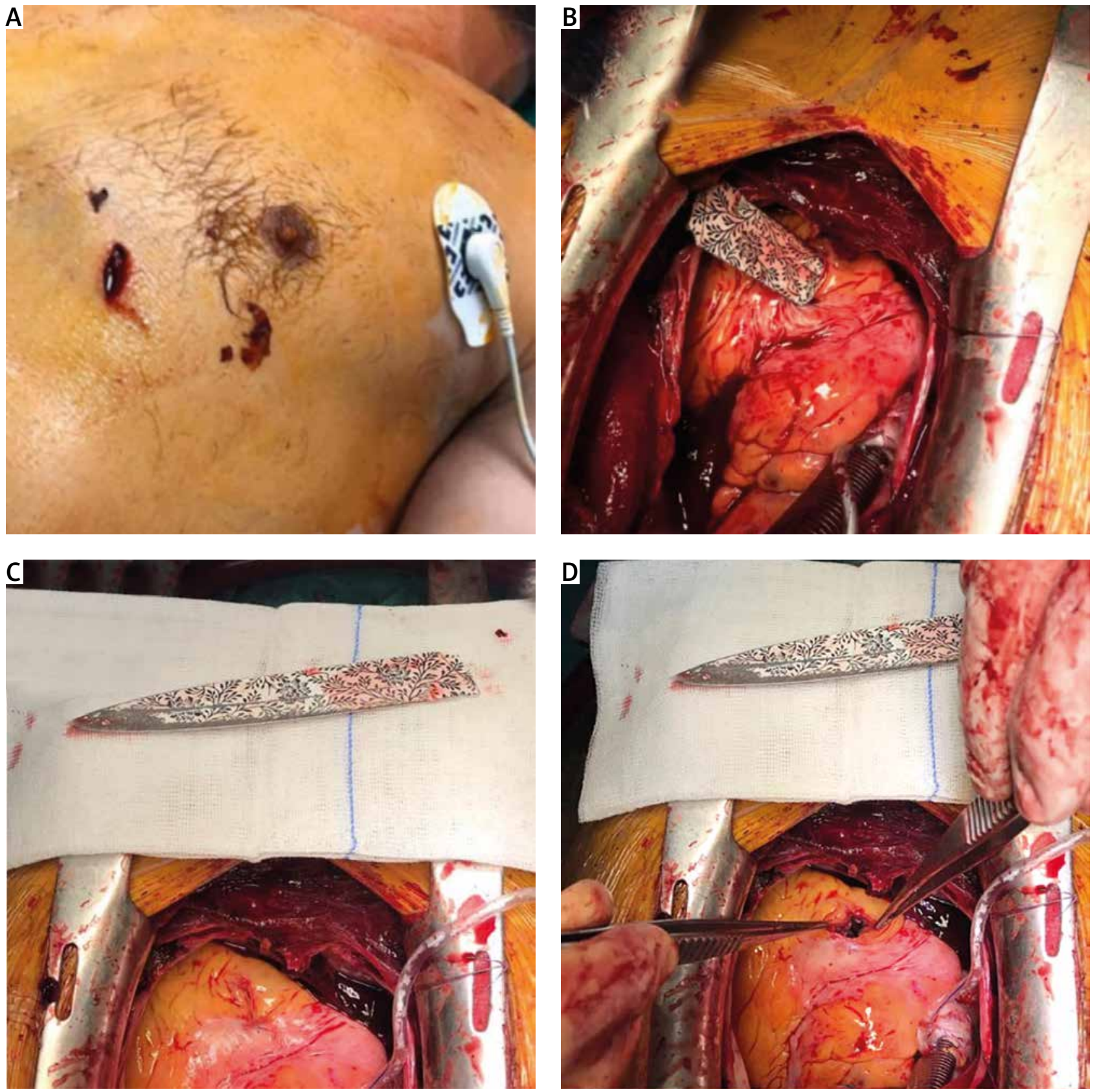

Figure 1. A - External view of the wound in the patient prepared for surgery. B - Condition after thoracotomy. View through the pericardial window on the blade causing penetrating cardiac trauma. $\mathbf{C}$ - The image showing the whole element evacuated from the patient's heart. Notably, nearly $2 / 7$ of the blade was buried in the cardiac muscle. D - Additionally the surgeon revealed a stab wound created by a knife blade

culation (ECC) with normothermia and blood cardioplegia. A flower-decorated ceramic kitchen knife blade with no visible handle was found stuck in situ in the heart (Figure 1). A transesophageal echocardiogram (TEE) was performed during surgery and showed the $1.2 \mathrm{~cm}$ patent foramen ovale (PFO) with severe tricuspid regurgitation. The blade was removed from the right ventricle. The dissected tricuspid valve leaflets and papillary muscles were observed from the right atrial approach. Due to the crushing of the subvalvular apparatus, replacement of the tricuspid valve with a Carpentier-Edwards Magna $29 \mathrm{~mm}$ mitral bioprosthesis had to be performed. The PFO was closed with a contin- uous suture. An epicardial electrode was implanted. The closure procedures were performed surgically. On the day after surgery, follow-up ECHO showed fluid, mainly behind the right atrium (fluid size up to $11 \mathrm{~mm}$ ) with no signs of right ventricular collapse or pericardial tamponade. The size of the cardiac cavities was normal with a left ventricular ejection fraction (LVEF) of approximately 65\%. During postoperative care, the patient required decompression of the left pleural cavity due to iatrogenic pneumothorax and received group-compatible foreign blood. Pharmacological treatment was administered. At 12 days after surgery, the patient was classified as COVID-19 positive and further care 
proceeded with adherence to the isolation regime. During the rest of the hospital stay, the patient was conscious, and respiratory and hemodynamically stable with normal diuresis. The patient was discharged from the hospital 37 days after admission.

According to a study by Novakov [7], knives are the most popular weapon for stabbing injuries in the chest area, with sharp-edged knives being commonly used in homicides and suicides. As recommended [3, 5], a ventilatory and circulatory stable patient should be considered for conservative therapy especially in the COVID era. Nevertheless, the man reported a stabbing acute chest pain, which directed reasonable suspicion to a deeper injury. Initially, the suspicion was confirmed by blood tests. Taking imaging studies into account, it was decided to proceed with surgical treatment. The $2 \mathrm{~cm}$ wound was not just a superficial injury, but turned out to be an entry point through which the blade had penetrated deep into the thorax and affected the myocardium. The knife blade had sunk completely beneath the layers of skin and muscle. Interestingly, since it was not visible externally, the presence of the nonmetallic ceramic foreign body could be confirmed by CXR. Groedel's heart-to-lung ratio was increased in this case [5], representing a combination of both cardiac tamponade and reduction of the thoracic cavity. An explanation of the role of tamponade can be found in the work of Naclerio et al. [5], who elaborate on the various mechanisms of decreased cardiac output and its consequences, both fatal and life-saving, noting that tamponade, through its effect of exerting pressure on the bleeding heart wall, played an important role in controlling hemorrhage and allowed the majority of these patients to reach the hospital alive [5]. We show in our study that the role of the tamponade was additionally taken over by the knife blade, which exerted pressure and at the same time acted as a wound closure to prevent the formation of tamponade. Removal of the blade would result in massive bleeding and hemodynamic collapse, so the patient was referred for surgery and subsequently connected to the ECC. The crucial point of surgery is the time at which cardiac compression is released. An oversight could lead to both subsequent clot formation and sudden pressure fluctuations. The ventricular location of the injury in the thick walls with great contractile force favored hemostasis and closure of the wound [5]. Mechanical closure by the blade prevented collapse of the right ventricle. The primary cause of the penetrating chest wound may negate the initial medical questioning and should broaden the suspicion towards an assassination or suicide attempt. In our case, the patient lied from the beginning and was aware of being stabbed with a knife, which could have had fatal consequences.

In conclusion, the presence of cardiac tamponade in the patient with penetrating cardiac trauma should always be considered and surgical management must be demonstrated according to the patient's general condition. Because of the possibility of SARS-CoV-2 infection, hospitalization after penetrating cardiac trauma must be done cautiously to minimize the risk of complications and not prolong recovery time.

\section{Disclosure}

The authors report no conflict of interest.

\section{References}

1. Pereira BMT, Nogueira VB, Calderan TRA, Villaça MP, Petrucci O, Fraga GP. Penetrating cardiac trauma: 20-y experience from a university teaching hospital. J Surg Res 2013; 183: 792-797.

2. Khan HAR, Gilani JA, Pervez M Bin, Hashmi S, Hasan S. Penetrating cardiac trauma: a retrospective case series from Karachi. J Pak Med Assoc 2018; 68: 1285-1287.

3. Kaljusto ML, Skaga NO, Pillgram-Larsen J, Tønnessen T. Survival predictor for penetrating cardiac injury; a 10-year consecutive cohort from a Scandinavian trauma center. Scand J Trauma Resusc Emerg Med 2015; 23: 41-48.

4. Campbell NC, Thomson SR, Muckart DJ, Meumann CM, Van Middelkoop I, Botha JB. Review of 1198 cases of penetrating cardiac trauma. Br J Surg 1997; 84: $1737-1740$.

5. Naclerio EA. Penetrating wounds of the heart: experience with 249 patients. Dis Chest 1964; 46: 19-22.

6. Furukawa H, Tsuchiya K, Ogata K, Kabuto Y, lida Y. Penetrating knife injury to the heart. Jpn J Thorac Cardiovasc Surg 2000; 48: 142-144.

7. Novakov IP. Clinics in surgery thoracic stab wound: a curious case report. Clin Surg 2018; 3: 2183. 